\title{
AVALIAÇÃO TARDIA DE DOENTES GASTRECTOMIZADOS POR ÚLCERA PÉPTICA: aspectos clínicos, endoscópicos e histopatológicos
}

\author{
João de Souza COELHO-NETO, Nelson Adami ANDREOLLO, Luiz Roberto LOPES, \\ Nancy F. NISHIMURA, Nelson Ary BRANDALISE e Luiz Sergio LEONARDI
}

RESUMO - Racional - Atualmente são raras as vezes em que é necessária a realização de uma gastrectomia, já que os inibidores da bomba de prótons associados aos esquemas antibióticos usados para erradicar o Helicobacter pylori, mudaram o enfoque do tratamento da úlcera péptica. Objetivos - Avaliar tardiamente os doentes submetidos a gastrectomia parcial no tratamento da úlcera péptica, em época em que o Helicobacter pylori ainda não era erradicado de forma intencional, incluindo os sintomas pós-operatórios em comparação com os pré-operatórios e a incidência de síndromes pós-gastrectomias; avaliação endoscópica, incluindo o tipo de cirurgia realizada e os achados macroscópicos da mucosa do coto gástrico, duodeno ou jejuno, dependendo do tipo de reconstrução; avaliação histopatológica, incluindo a pesquisa da bactéria por dois métodos: histológico e teste de urease; e sua possível associação presente no estômago remanescente com as avaliações clínica, endoscópica e histopatológica pósoperatórias. Casuística e Métodos - Cinqüenta e nove doentes gastrectomizados por úlcera péptica entre os anos de 1985 e 1993 foram avaliados, sendo que 44 (74,6\%) eram do sexo masculino e tinham idade média de 55 anos, com variação de 31 a 77 anos, passaram por entrevista clínica e por exame endoscópico. O Helicobacter pylori foi pesquisado nas peças cirúrgicas ressecadas, para constatação da sua presença ou não no pré-operatório. Resultados - A avaliação clínica no pós-operatório tardio mostrou que $96 \%$ dos doentes apresentaram excelentes e bons resultados (Visick I e II). Os sintomas pós-operatórios mais comuns foram dispepsia leve e outras queixas, como diarréia, anemia e dumping, que ocorreram, respectivamente, em 11 (18,6\%), 2 (3,4\%) e 2 $(3,4 \%)$ casos. A reconstrução tipo Billroth I trouxe melhores resultados clínicos tardios, quando comparada com as reconstruções tipo Billroth II e Y-de-Roux. Na avaliação endoscópica, a maioria dos doentes $(52,5 \%)$ apresentou exame normal, enquanto que os demais apresentaram gastrites enantematosa $(37,3 \%)$ e erosiva (8,5\%). Recidiva ulcerosa ocorreu em dois doentes $(3,4 \%)$. Na análise histopatológica, foi observada incidência elevada de gastrite crônica (98,3\%). A presença de Helicobacter pylori ocorreu em $86 \%$ dos doentes antes da cirurgia e em $89,8 \%$ no pós-operatório tardio. Conclusões - Os doentes gastrectomizados apresentaram boa evolução clínica pós-operatória tardia. A gastrectomia parcial com reconstrução tipo Billroth I foi a que trouxe melhores resultados clínicos a longo prazo. O Helicobacter pylori, apesar de estar presente no coto gástrico em $89,8 \%$ dos casos, não influenciou de forma negativa nos resultados clínicos, endoscópicos ou histopatológicos no pós-operatório tardio.

DESCRITORES - Úlcera péptica. Gastrectomia. Helicobacter pylori. Síndromes pós-gastrectomia.

\section{INTRODUÇÃO}

Os novos medicamentos que inibem a produção de ácido pelo estômago e os novos conhecimentos sobre o Helicobacter pylori (HP) mudaram muito o enfoque do tratamento da úlcera péptica. Atualmente são raras as vezes em que é necessária a realização de uma gastrectomia, sendo exceções as operações para as complicações das úlceras, como as obstruções, hemorragias e perfurações. Os potentes inibidores das bombas de prótons associados aos esquemas antibióticos usados para erradicar o HP são capazes de levar à cura da úlcera péptica ${ }^{(12,22,25,39,40)}$.

Não há na literatura muitos trabalhos relatando a avaliação ampla tardia de doentes gastrectomizados e sua relação com o HP, bem como a recidiva ulcerosa, sintomatologia pós-operatória tardia, síndromes pós-gastrectomia, além de métodos diagnósticos e sua eficiência. É justamente o intuito de saber o que ocorre com os doentes gastrectomizados na era do HP que estimulou a realização deste estudo na forma mais abrangente possível.

\footnotetext{
Departamento de Cirurgia e Centro de Diagnóstico de Doenças do Aparelho Digestivo - GASTROCENTRO, Faculdade de Ciências Médicas da Universidade Estadual de Campinas - UNICAMP - Campinas, SP.

Endereço para correspondência: Dr. Nelson Adami Andreollo - Rua Francisco Humberto Zuppi, 1234 - Cidade Universitária - 13083-350 - Campinas, SP. E-mail: nandreollo@terra.com.br
} 
Os objetivos desta pesquisa foram avaliação clínica, endoscópica e histopatológica tardia, de doentes gastrectomizados por úlcera péptica.

\section{CASUÍSTICA E MÉTODO}

Este estudo foi aprovado pela Comissão de Ética Médica do Hospital das Clínicas da Universidade Estadual de Campinas - UNICAMP em 6/2/1998, parecer no 007/98.

Foram incluídos na pesquisa doentes previamente submetidos a gastrectomia parcial como tratamento da úlcera péptica, que tivessem um mínimo de 5 anos de seguimento pós-operatório, e que não tivessem recebido tratamento prévio para HP. A indicação cirúrgica em todos os casos foi por intratabilidade clínica, sendo portanto excluídos os doentes com complicações.

Dentre 156 doentes gastrectomizados no Grupo de Cirurgia de Esôfago, Estômago e Duodeno, no período de fevereiro de 1985 a agosto de 1993 e convocados pelo serviço social do Hospital das Clínicas da UNICAMP, foram encontrados 61, sendo que 2 se recusaram a participar do presente estudo, restando 59.

No período de 14/5/1997 a 3/11/1999 os 59 doentes foram avaliados clinicamente e examinados através de endoscopia digestiva alta (EDA). O intervalo de tempo entre o tratamento cirúrgico e o controle clínico endoscópico foi, em média, de 96 meses, variando entre o tempo mínimo de 67 e o máximo de 177 meses. Eram 15 doentes $(25,4 \%)$ do sexo feminino e $44(74,6 \%)$ do sexo masculino, $54(91,5 \%)$ eram brancos e $5(8,5 \%)$ eram negros. Na época da cirurgia a idade variou de 31 a 77 anos sendo a média de 55,5 anos.

Para facilitar a análise estatística com relação ao local das úlceras, os achados foram divididos em três grandes grupos, conforme adaptação à classificação de Johnson ${ }^{(23)}$, de forma a avaliar em um único grupo as úlceras que possuem etiopatogenia semelhante. Desta forma, as úlceras pilóricas, quando referidas, foram agrupadas às úlceras duodenais, constituindo um dos três grupos; as úlceras gástricas constituíram o segundo e os doentes que apresentavam úlceras gástricas e duodenais o terceiro grupo.

A localização das úlceras no momento do diagnóstico préoperatório foi demonstrada através da EDA, sendo: 28 doentes com úlcera duodenal (UD - 47,5\%), 18 doentes com úlcera gástrica (UG - 30,5\%) e a associação entre úlcera gástrica e duodenal foi observada em 13 doentes (UG + UD - 22\%).

Todos os 59 pacientes foram submetidos a gastrectomia, conforme técnicas cirúrgicas padronizadas - técnica de Schoemaker à Billroth $\mathrm{I}(\mathrm{BI})$ e técnica de Hofmeister à Billroth II $(\mathrm{BII})^{(34)}$, tendo sido realizadas por um único grupo de cirurgiões do Hospital das Clínicas - UNICAMP. A Tabela 1 demonstra o tipo de reconstrução utilizada, a realização ou não de vagotomia troncular (VT), em relação ao local das úlceras.

TABELA 1 - Tipo de reconstrução utilizada e local da úlcera

\begin{tabular}{lccccc}
\hline Local da úlcera & BI + VT & BI & BII + VT & BII & $\begin{array}{c}\text { Y-Roux + } \\
\text { VT }\end{array}$ \\
\hline UD (28) & 21 & - & & 4 & 3 \\
UG (18) & - & 16 & - & - \\
UG + UD (13) & 11 & - & & - & 2 \\
\hline
\end{tabular}

\section{Protocolo e entrevista clínica}

No período de 14/5/1997 a 3/11/1999, todos os 59 doentes que concordaram em participar da pesquisa e assinaram o termo de consentimento informado foram entrevistados pelo autor, seguindo o roteiro de perguntas dirigidas pré-estabelecidas. Todos foram informados e esclarecidos sobre o estudo e seus objetivos, concordando em realizar EDA de controle e as biopsias.

Os dados obtidos com relação às condições clínicas e resultados de evolução cirúrgica foram avaliados conforme a classificação de Visick $^{(42)}$ modificada por GOLIGHER et al. ${ }^{(13)}$

\section{Endoscopia digestiva alta e biopsias}

A EDA foi realizada em todos os casos, conforme preparo padronizado pelo Serviço de Endoscopia Digestiva do GASTROCENTRO. Os achados endoscópicos foram classificados segundo os critérios de Sydney ${ }^{(10)}$.

Durante a endoscopia, foram coletados cinco fragmentos de mucosa, sendo, respectivamente: parede anterior e posterior do corpo gástrico para análise histopatológica e pesquisa do HP, parede anterior e posterior do corpo e do fundo gástrico para o teste da urease. Além disso, caso fosse encontrada alguma alteração endoscópica na mucosa, gástrica, duodenal, jejunal ou de anastomose, outra biopsia dirigida era feita para análise histopatológica ${ }^{(23,28)}$.

Os resultados do teste de urease dos três fragmentos de cada doente foram anotados como positivo, negativo ou inconclusivo para a presença do HP. Considerou-se como positivo aquele doente que tivesse a bactéria em, pelo menos, um dos cinco fragmentos.

$\mathrm{O}$ estudo histopatológico das biopsias endoscópicas foi analisado por um patologista experiente em doenças digestivas, usando-se a coloração hematoxilina-eosina e em alguns casos, também a coloração de Giemsa. Foi considerado positivo para a presença da bactéria HP quando as mesmas foram encontradas e identificadas, sem margem de dúvida ${ }^{(23,28)}$.

Foi realizada revisão das lâminas das peças cirúrgicas da época da gastrectomia, para verificar as alterações presentes no estômago naquela ocasião e se havia ou não a presença de HP antes da cirurgia, uma vez que nenhum dos doentes tinha recebido medicação para a sua erradicação.

Foi realizada análise estatística com o objetivo de possibilitar a análise descritiva das variáveis pré-operatórias, pós-operatórias e da anatomia patológica e comparar as variáveis de interesse, utilizando tabelas de freqüências para as variáveis categóricas e estatísticas descritivas para a variável contínua. $\mathrm{O}$ teste exato de Fisher foi empregado para verificar se existe associação ou diferença entre as variáveis categóricas $(P \leq 0,05)$.

\section{RESULTADOS}

\section{Sintomas pós-operatórios e restrições alimentares}

A classificação de Visick foi utilizada para caracterizar a intensidade dos sintomas pós-operatórios, sendo considerado como Visick I aqueles totalmente assintomáticos, Visick II com sintomas leves, Visick III com sintomas moderados e Visick IV com sintomas intensos. A Tabela 2 mostra o número de doentes em relação a essa classificação. 
TABELA 2 - Classificação de Visick

\begin{tabular}{ccc}
\hline Visick & $\mathbf{n}^{\circ}$ de doentes & $\%$ \\
\hline I & 33 & 55,9 \\
II & 24 & 40,7 \\
III & 2 & 3,4 \\
IV & - & - \\
\hline
\end{tabular}

A maioria dos doentes apresentou ganho de peso no período pós-operatório $(40 / 59=67,8 \%), 14$ doentes não alteraram o peso $(14 / 59=23,7 \%)$ e apenas 5 apresentaram perda de peso $(5 / 59=$ $8,5 \%$ ). Destes cinco doentes que perderam peso, três apresentavam reconstrução do tipo Y-de-Roux e dois com BI.

Quanto à análise das restrições alimentares no pós-operatório, o grupo sem restrições totalizou 48 doentes $(81,4 \%)$, sendo que destes, $42(42 / 48=87,5 \%)$ tinham reconstrução a BI $(P<0,05)$.

Dentre os sintomas pós-operatórios analisados, a diarréia ocorreu em 11 doentes $(11 / 59=18,6 \%)$, sendo diarréia eventual em 8 doentes e crônica em 3 , a anemia estava presente em 2 doentes $(2 / 59=3,4 \%)$ e sintomatologia compatível com dumping em 2 doentes $(2 / 59=3,4 \%)$.

\section{Helicobacter pylori na peça cirúrgica e na biopsia endoscópica pós-operatória}

As lâminas das peças cirúrgicas ressecadas foram reavaliadas e, então, realizada a pesquisa do HP ou em alguns casos, foram feitas novas lâminas, empregando-se o bloco de parafina arquivado. Assim, em 57 casos, foi possível pela revisão das lâminas, determinar a presença ou não do HP.

No pós-operatório foi verificada a presença ou não do HP pelo exame histopatológico das biopsias endoscópicas e teste de urease (dois de histologia e três de urease). A Tabela 3 mostra a ocorrência do HP nas peças cirúrgicas, em relação ao local das úlceras e nas biopsias pós-operatórias (PO).

TABELA 3 - Ocorrência do HP e respectivas percentagens

\begin{tabular}{lcc}
\hline Local & $\mathrm{HP}+$ & HP- \\
\hline Peça cirúrgica & $49(86 \%)$ & $8(14 \%)$ \\
UD (28) & $33(82 \%)$ & $5(18 \%)$ \\
UG (18) & $16(88 \%)$ & $2(12 \%)$ \\
UG + UD (13) & $10(76 \%)$ & $3(24 \%)$ \\
Biopsia PO & $53(89,8 \%)$ & $6(11,2 \%)$ \\
\hline
\end{tabular}

Quando cada indivíduo foi analisado isoladamente, verificouse que dos 49 doentes positivos para HP antes da gastrectomia, 46 se mantiveram positivos $(46 / 49=93,8 \%$ dos previamente positivos) no pós-operatório tardiamente; dos oito negativos para HP antes da cirurgia, apenas dois se mantiveram negativos $(2 / 8=25 \%$ dos previamente negativos); seis eram negativos e se tornaram positivos $(6 / 8=75 \%$ dos previamente negativos $)$ e três que eram positivos, não têm mais o HP no momento (3/49 $=6,2 \%$ dos previamente positivos). Não foi possível essa avaliação em dois casos.

\section{Sintomas versus Helicobacter pylori}

Avaliando-se a relação entre a presença de HP nas biopsias e presença de sintomas pós-operatórios, observou-se que dos 26 doentes que tinham algum tipo de sintoma, $25(25 / 26=96,1 \%)$ eram HP positivo. Dos 33 assintomáticos, 85\% (28/33) eram HP positivo e apenas $5(5 / 33=15,5 \%)$ eram HP negativo. Dentre os 53 doentes HP positivo, apenas $25(25 / 53=47,2 \%)$ eram sintomáticos. Não houve significância estatística na comparação dos grupos $(P>0,05)$.

Especificamente para a diarréia, observou-se que dos 11 doentes com este sintoma, 100\% deles eram HP positivo; por outro lado, dos 48 doentes sem diarréia, $42(42 / 48=87,5 \%)$ também eram HP positivo, portanto não houve diferença estatística $(P>0,05)$.

Quanto à anemia e à síndrome de dumping também não houve diferença estatística com relação à presença ou não de HP $(P>0,05)$.

$\mathrm{Na}$ comparação entre a presença do HP nas biopsias e a classificação de Visick também não houve diferença estatística $(P>0,05)$. A Tabela 4 mostra a ocorrência de HP em relação à classificação de Visick.

TABELA 4 - Ocorrência de HP e a classificação de Visick

\begin{tabular}{ccc}
\hline Visick & HP+ & HP- \\
\hline I (32) & $28(87,5 \%)$ & $4(12,5 \%)$ \\
II (25) & $24(96 \%)$ & $1(4 \%)$ \\
III (2) & $1(50 \%)$ & $1(50 \%)$ \\
IV (0) & - & - \\
\hline
\end{tabular}

\section{Achados endoscópicos}

$\mathrm{Na}$ EDA verificou-se que a mucosa do coto gástrico apresentavase normal na maioria dos doentes, ou seja, em 31 deles $(31 / 59=$ $52,5 \%$ ), a gastrite endoscópica enantematosa foi diagnosticada em 22 doentes $(22 / 59=37,3 \%)$ e gastrite endoscópica erosiva em 5 doentes $(5 / 59=8,5 \%)$.

Quanto à recidiva pós-operatória de úlcera péptica, três doentes informaram história clínica compatível com essa doença. Desses casos, um teve quadro bem documentado de úlcera de boca anastomótica 2 anos e 8 meses após a cirurgia; outro teve quadro de hemorragia digestiva alta 3 meses antes da endoscopia deste estudo e a endoscopia feita na ocasião constatou úlcera, sendo que esse doente recebeu inclusive tratamento para o HP; e um terceiro doente teve quadro de hemorragia digestiva alta 11 meses após este estudo e à endoscopia foi vista úlcera duodenal. Entretanto, durante a realização da EDA para este estudo, foi constatada a presença de úlcera ativa em outros dois doentes $(2 / 59=3,4 \%)$. Assim, ao final foi considerada recidiva ulcerosa pós-operatória no total de cinco doentes $(5 / 59=8,5 \%)$.

Além disso, analisou-se se os achados endoscópicos tinham alguma relação com a presença do HP. Foi observado que a presença ou ausência do HP no coto gástrico não foi estatisticamente significante para os diversos achados endoscópicos. Dos 31 doentes com endoscopia normal, $27(27 / 31=87,1 \%)$ eram HP positivo, porém dos 28 doentes com alguma alteração endoscópica, $26(26 / 28=92,9 \%)$ também eram HP positivo $(P>0,05)$. Os 2 doentes com úlcera péptica diagnosticada durante o exame endoscópico apresentavam HP positivo (100\%), por outro lado, dentre os 57 que não tinham úlcera, $89,3 \%$ também eram HP positivo $(P>0,05)$. Quanto à presença de gastrites endoscópica enantematosa e erosiva, o mesmo resultado foi verificado, ou seja, o HP positivo não influenciou nos resultados. 
Comparando a presença de bile e o tipo de reconstrução cirúrgica, foi observado que dos 24 doentes com bile no coto gástrico, 19 tinham reconstrução tipo BI (39,6\% dos BI), 4 tinham reconstrução tipo BII $(66,6 \%$ dos BII) e 1 com reconstrução tipo Y-de-Roux (20\% dos Y-de-Roux).

\section{Resultados dos estudos histopatológicos} das biopsias endoscópicas

No estudo histopatológico dos fragmentos de biopsias gástricas obtidas durante a endoscopia observou-se gastrite crônica em 58 doentes $(58 / 59=98,3 \%)$ e apenas $1(1 / 59=1,7 \%)$ doente apresentava mucosa gástrica normal. A Tabela 5 mostra as alterações histopatológicas nas biopsias do coto gástrico remanescente.

TABELA 5 - Alterações histopatológicas das biopsias gástricas

\begin{tabular}{lcc}
\hline Biopsias gástricas & $\mathbf{n}^{\text {o de casos }}$ & $\%$ \\
\hline Normal & 1 & 1,7 \\
Gastrite crônica intensa & 3 & 5,2 \\
Gastrite crônica moderada & 27 & 46,5 \\
Gastrite crônica leve & 28 & 48,3 \\
Mucosa gástrica atrófica & 7 & 11,9 \\
Hiperplasia gástrica & 2 & 3,4 \\
Metaplasia intestinal & 13 & 22 \\
Displasia & 2 & 3,4 \\
\hline
\end{tabular}

Não foi observada influência estatisticamente significante com relação à presença do HP no coto gástrico sobre os estudos histopatológicos. No doente com mucosa normal à histologia, 0 HP era positivo (100\%) e nos 58 doentes com alguma alteração de mucosa o HP estava positivo em $89,7 \%(P>0,05)$.

\section{Resultados dos estudos histopatológicos}

das biopsias das peças cirúrgicas

Avaliando a relação entre a presença do HP no pré-operatório e os achados histopatológicos da peça cirúrgica, concluiu-se que não houve significado estatístico que pudesse correlacionar a presença do HP com algum achado histológico específico como gastrite crônica $(P=0.210)$, atrofia de mucosa gástrica $(P=1.000)$, hiperplasia, metaplasia $(P=0.705)$ ou mucosa normal $(P>0.05)$.

Sintomas pós-operatórios versus achados endoscópicos

Não houve diferença estatística entre a presença ou não de alterações endoscópicas e os sintomas no pós-operatório. Dos doentes com gastrite endoscópica enantematosa, 54,5\% eram sintomáticos e $45,5 \%$ eram assintomáticos $(P>0,05)$. Dos doentes com gastrite endoscópica erosiva, $40 \%$ eram assintomáticos e $60 \%$ eram sintomáticos $(P>0,05)$. Apesar de 31 doentes apresentarem a mucosa gástrica normal à endoscopia, 38,7\% deles apresentavam algum tipo de sintoma no pós-operatório e $61,3 \%$ eram assintomáticos $(P>0,05)$. Houve uma tendência à significância estatística quando foi avaliada a presença de úlcera à endoscopia, sendo que os dois doentes com recidiva de úlcera péptica eram sintomáticos $(P>0,05)$.

\section{Achados endoscópicos versus tipo de reconstrução cirúrgica}

Não houve correlação estatisticamente significante, porém houve tendência quando se observou que os doentes com reconstrução tipo BII têm mais gastrite endoscópica $(66,7 \%)$, do que os com reconstrução tipo BI $(37,5 \%)$ ou Y-de-Roux $(0 \%)$ $(P>0.05)$. Não foi verificada diferença estatística com relação à presença de gastrite endoscópica erosiva e tipo de reconstrução cirúrgica. Mucosa normal foi observada em 50\% dos doentes com BI, 33,3\% dos doentes com BII e em todos com reconstrução tipo Y-de-Roux. Portanto, houve tendência maior a apresentar mucosa normal à endoscopia quando se fez reconstrução tipo Y-de-Roux $(P>0.05)$. Os doentes em que foi diagnosticada úlcera na EDA tinham reconstrução tipo BI.

\section{DISCUSSÃO}

A maioria dos doentes foi submetida a gastrectomia parcial, e empregada a técnica clássica de Schoemaker, com reconstrução tipo $\mathrm{BI}$ associada ou não à vagotomia ${ }^{(34)}$, por ser mais fisiológica, com o duodeno no trânsito alimentar, menor risco de desenvolver câncer no coto gástrico e com melhores resultados a longo prazo ${ }^{(1,2,26,30)}$.

Os resultados encontrados nesta casuística são considerados satisfatórios e estão muito próximos aos da literatura. Trinta e três doentes $(55,9 \%)$ dos 59 estão absolutamente assintomáticos. Pela classificação de Visick os resultados também são considerados muito bons, uma vez que foi considerado Visick I em 54,2\% e Visick II em 42,4\%, portanto, totalizando 96,5\% de doentes com Visick I e II. A positividade do HP na maioria deles no pósoperatório tardio não teve influência negativa nos resultados e na classificação do Visick.

SEZIN et al. ${ }^{(32)}$ revisando 710 doentes submetidos a gastrectomia para tratamento de úlcera péptica obteve excelentes e bons resultados (Visick I e II) em 94\%. Já TYTGAT et al. ${ }^{(41)}$, mencionam que doentes com Visick III e IV são esperados em cerca de 15 e $25 \%$ de doentes submetidos a vagotomia com drenagem gástrica e também a gastrectomia parcial como tratamento de doenças benignas.

PAPINI-BERTO ${ }^{(27)}$ estudando 71 doentes gastrectomizados, sendo 53 com gastrectomia parcial e $18 \mathrm{com}$ gastrectomia total e sendo a maioria $(68 \%)$ por úlcera péptica, verificou perda de peso referida em $70 \%$ dos doentes num período pós-operatório variando de 6 a 60 meses e que esta ocorreu devido à redução e mudança de alguns hábitos alimentares.

Ainda com relação aos sintomas pós-operatórios, verificou-se que a presença do HP na maioria dos doentes não teve influência negativa, ou seja, não houve diferença estatística mostrando que a presença do HP no coto gástrico remanescente possa causar mais sintomas. Dos 33 doentes assintomáticos, 28 (84,5\%) eram HP positivo e dos 26 doentes com algum tipo de sintoma pósoperatório, $25(96,1 \%)$ também eram HP positivo.

A taxa de recidiva ulcerosa ocorre em cerca de $2 \%$ a $3 \%$ dos doentes operados. $\mathrm{Na}$ antrectomia onde se resseca cerca de $40 \%$ do estômago distal, associada à vagotomia, a taxa de recidiva é em torno de $0,6 \%{ }^{(2,19,31,32,35)}$ JUNCOSA et al. ${ }^{(16)}$, revendo 1200 doentes operados por úlcera péptica gastroduodenal após 10 e 15 anos, observaram recidiva ulcerosa em $6,6 \%$ deles. Já um grupo argentino observou $1,8 \%$ de recidiva em estudo de revisão de 710 doentes submetidos a ressecção gástrica para tratar úlcera ${ }^{(32)}$. TYTGAT et al. ${ }^{(41)}$ demonstram que há um aumento linear de 
recorrência ulcerosa no decorrer dos anos e que a taxa de recidiva após antrectomia com vagotomia troncular é de cerca de 1 a $5 \%$. ARCHIMANDRITIS et al. ${ }^{(3)}$, revendo 80 doentes gastrectomizados por doença benigna observaram recidiva ulcerosa de $14 \%$.

A taxa de recidiva verificada nesta pesquisa totalizou 8,5\% (cinco doentes), porém no momento do estudo a úlcera ativa recidivada foi diagnosticada em apenas 3,4\% (dois doentes). Provavelmente foi verificada a recidiva ulcerosa nesta taxa porque foi realizada EDA em todos os casos, mesmo que os doentes estivessem assintomáticos. Os dois doentes diagnosticados com úlcera estavam oligossintomáticos, referindo apenas sintomas dispépticos leves.

Todos os doentes deste estudo com recidiva ulcerosa eram HP positivo no coto gástrico, porém nem sempre este fato tem sido relatado na literatura. LEE et al. ${ }^{(11)}$ e LEIVONEN et al.(21), consideraram que apesar da persistência do HP no pós-operatório de gastrectomias, ela não pode ser considerada como fator absoluto de causa de recidiva ulcerosa no coto gástrico ou na anastomose.

Quando for necessária a pesquisa do HP no coto gástrico remanescente pós-gastrectomia é importante e indispensável a coleta de vários fragmentos de mucosa e, se possível, a utilização de mais de um método diagnóstico, minimizando assim a chance de não se conseguir diagnosticar a infecção. É importante também a coleta de fragmentos de todas as paredes do estômago, anterior, posterior e do fundo gástrico.

A maioria das síndromes pós-gastrectomia resulta de distúrbios da motilidade gástrica ${ }^{(9,11,31)}$. Sua ocorrência se situa entre $5 \%$ e $50 \%$, mas na maioria das publicações sua incidência é próxima de $20 \%{ }^{(7,36)}$. O sexo também pode influenciar, sendo sua prevalência mais elevada nos doentes do sexo feminino ${ }^{(18,39)}$. Apenas cerca de $1 \%$ dos doentes que desenvolvem alguma síndrome pósgastrectomia apresentam este distúrbio permanentemente e requerem algum tratamento cirúrgico ${ }^{(9)}$.

A diarréia pós-operatória é uma das complicações freqüentes após cirurgia para úlcera péptica, cuja freqüência pode variar de $2 \%$ a $30 \%$, dependendo da técnica cirúrgica utilizada ${ }^{(6,9)}$. BRAGHETTO et al. ${ }^{(6)}$ analisando 519 doentes operados, verificaram que dos 227 doentes operados por úlcera gástrica, $35(15,4 \%)$ tiveram diarréia no pós-operatório, sendo $2(0,9 \%)$ diarréia severa e $33(14,6 \%)$ diarréia leve, e que a maioria deles foi submetida a ressecção gástrica extensa; e dos 292 operados por úlcera duodenal, 34 (11,6\%) tiveram diarréia, sendo 17 (6,2\%) leve, 14 (4,8\%) moderada e $2(0,2 \%)$ diarréia severa, principalmente quando submetidos a vagotomia troncular mais antrectomia. A maioria dos doentes responde bem ao tratamento clínico com dieta, antidiarréicos e antiespasmódicos. Este estudo constatou 11 doentes $(18,6 \%)$ com diarréia pós-gastrectomia, sendo diarréia temporária em 8 e crônica em 3 .

A síndrome de dumping tem sido relacionada com a velocidade de esvaziamento gástrico depois da gastrectomia com vagotomia troncular. A reconstrução a BII resulta habitualmente em esvaziamento gástrico mais rápido por não ter o mecanismo do piloro e nem o do duodeno, e com isso a incidência de dumping pode chegar a $50 \%$ na fase pós-operatória precoce ${ }^{(7}$, 9). A reconstrução tipo $\mathrm{BI}$, por ter a resistência do duodeno no trânsito alimentar, causa menos dumping. Já a reconstrução tipo Y-de-Roux costuma levar a um esvaziamento gástrico lento e praticamente não resulta em dumping ${ }^{(31,41)}$.
A síndrome de dumping teve baixa incidência nesta casuística, estando presente em dois doentes do sexo feminino $(3,4 \%)$ e coincidentemente submetidos a gastrectomia tipo BI. OKUMURA et al. ${ }^{(24)}$ também verificaram maior incidência de síndrome de dumping em mulheres.

A anemia pós-gastrectomia quase sempre é do tipo microcítica e ocorre em cerca de $20 \%$ dos doentes ${ }^{(38)}$. TOVEY e CLARK ${ }^{(38)}$ estudaram as causas carenciais e os tratamentos para a anemia pós-gastrectomia. Nesta casuística foi observada anemia em apenas dois $(3,4 \%)$ doentes. Porém, não foi feita nenhuma pesquisa laboratorial adicional por não ter sido este o objetivo do trabalho.

Analisando a ocorrência das síndromes pós-gastrectomia nesta pesquisa, também se verificou que o HP positivo não influenciou no aumento de sua ocorrência e nem das suas manifestações clínicas.

Ainda há controvérsias na literatura internacional no que diz respeito à presença de HP no coto gástrico remanescente pós-gastrectomia parcial e se está indicado o seu tratamento ${ }^{(4,}$ $5,8,20,25,29,37)$. É sabido que o antro é a localização preferencial para a permanência do HP e é justamente esta a região do estômago ressecada na gastrectomia parcial (distal) indicada no tratamento da úlcera péptica. Portanto, seria possível a cirurgia de ressecção gástrica suficiente para erradicar o HP? Esta pesquisa conclui que não. Dos 59 doentes estudados, 53 deles $(89,8 \%)$ ainda apresentavam o HP no coto gástrico no pós-operatório tardio (média de 95,9 meses ou 8 anos de tempo de seguimento) detectado por estudo histopatológico ou pelo teste de urease.

A revisão sistemática de 36 estudos feita por DANESH et al. ${ }^{(8)}$, demonstrou que a prevalência de HP pós-gastrectomia foi de apenas 50\% (tempo médio de estudo pós-operatório de 10 anos). Os autores não tiveram evidências suficientes para comparar a incidência do HP para cada tipo de reconstrução cirúrgica como por exemplo, BI, BII ou Y-de-Roux. Este achado está em desacordo com os presentes resultados, cuja prevalência do HP foi muito maior. BAHNACY et al. ${ }^{(5)}$ verificaram a presença de HP no estômago remanescente em 50\% dos doentes gastrectomizados por úlcera péptica e incidência de 9,5\% nos doentes gastrectomizados por câncer gástrico. SHEU et al. ${ }^{(50)}$ obtiveram achados semelhantes com 52,3\% de infecção por HP em um grupo de 86 doentes em seguimento pós-gastrectomia.

Segundo DANESH et al. ${ }^{(8)}$ e KARAT \& GRIFFIN ${ }^{(17)}$ esta baixa prevalência de HP pós-gastrectomia parcial se deve parcialmente à remoção do antro gástrico e parcialmente ao efeito bactericida do refluxo biliar prolongado para o coto gástrico. No estudo presente, foi observada bile no coto gástrico de 24 doentes (40,7\%) e nem por isto foi verificada baixa ocorrência de HP neste grupo. Além disso, foi constatado HP positivo em 91,7\% dos doentes com gastrectomia com reconstrução BI, em $60 \%$ dos doentes com reconstrução em Y-de-Roux e em 100\% dos doentes com reconstrução BII, o oposto do observado em grande parte da literatura. Por que alguns doentes que tinham HP no pré-operatório deixaram de tê-lo no pós-operatório tardio? HEYMANN e WILSON ${ }^{(14)}$ afirmam que muitos doentes fazem uso de antibióticos (terapêutico ou profilático) durante o período de internação perioperatória e isto poderia eventualmente 
erradicar o HP. Outro aspecto discutível é a condição socioeconômica dos doentes desta série que, por ser de um modo geral baixa, tenha contribuído na persistência do HP no pós-operatório.

Concluindo, alguns trabalhos mostram que a presença do HP no coto gástrico remanescente é comum, porém sua incidência é menor do que antes da cirurgia ${ }^{(5,33)}$. Neste estudo, $86 \%$ dos doentes tinham HP no pré-operatório e $89,8 \%$ deles também o tinham no pós-operatório. Portanto, o HP incidiu mais no coto gástrico remanescente do que no período préoperatório, porém sem diferença estatística. E dos 49 doentes que tinham HP no pré-operatório, 46 se mantiveram positivos ( $93,8 \%$ deles) e mais 6 se tornaram positivos no decorrer destes anos de seguimento.

A avaliação por EDA de todos os doentes no pós-operatório tardio permitiu uma completa visão da situação macroscópica do coto gástrico. Os achados mostraram mucosa gástrica normal à visão endoscópica na maioria dos casos $(52,5 \%)$. Gastrite endoscópica enantematosa foi observada em $22(37,3 \%)$ e gastrite endoscópica erosiva em 5 doentes $(8,5 \%)$. Úlcera ativa durante a EDA foi verificada em dois doentes, como já comentado anteriormente. Mais uma vez a positividade de HP no coto gástrico não influenciou na ocorrência dos achados, pois estava presente tanto em $87,1 \%$ dos doentes com mucosa normal, como em $92,2 \%$ dos doentes com alguma alteração da mucosa gástrica $(P<0,005)$. Os doentes com gastrectomia com reconstrução tipo BII tiveram maior tendência a apresentar gastrite endoscópica $(66,7 \%)$ do que os com reconstrução tipo BI $(37,5 \%)$ e Y-de-Roux $(0 \%)$. Todos os doentes com Y-de-Roux tinham mucosa gástrica normal à EDA, seguidos dos doentes com BI (50\% deles).

\section{CONCLUSÕES}

A análise final dos resultados mostrou que o tratamento cirúrgico proposto para este grupo de doentes trouxe resultados tardios muito satisfatórios. A hemigastrectomia, seguida de vagotomia troncular ou não, contribuiu para diminuir as recidivas ulcerosas. A reconstrução cirúrgica do tipo BI também mostrou freqüência pequena de sintomas tardios. O Helicobacter pylori apesar de estar presente no coto gástrico em $89,8 \%$ dos doentes, não influenciou de forma negativa nos resultados clínicos, endoscópicos ou histopatológicos no pós-operatório tardio.

\section{AGRADECIMENTOS}

Ao Dr. José Augusto G. Stedile, do Serviço de Anatomia Patológica do GASTROCENTRO - UNICAMP, pela valiosa colaboração na análise histopatológica.

Coelho-Neto JS, Andreollo NA, Lopes LR, Nishimura NF, Brandalise NA, Leonardi LS. Late follow-up of gastrectomized patients for peptic ulcer: clinical, endoscopic and histopathological aspects. Arq Gastroenterol 2005;42(3):146-52.

ABSTRACT - Background - The gastrectomy is an uncommon procedure because the proton bomb inhibitors associated to the antibiotic outlines used to eradicate the Helicobacter pylori changed the focus of the peptic ulcer treatment. Aims - Later evaluation on those patients who underwent partial gastrectomy as a treatment for peptic ulcer, at that time when any drug to eradicate the Helicobacter pylori was not used. The clinical evaluation included the late postoperative symptoms and postgastrectomy syndromes like dumping, diarrhea, alkaline gastritis and nutritional aspects. The upper digestive endoscopy analysed the surgery reconstruction and the gastric stump, the duodenum and the jejunum mucosa aspects. The histopathological evaluation included looking for Helicobacter pylori by using two different methods: histology and urease test. Casuistic and Methods - Fifty-nine patients, 44 (74.6\%) male, median age 55.5 years old (range from 31 to 77 years old), who underwent a clinical interview and an upper digestive endoscopy. Paraffin blocks from the surgical specimen were reviewed in order to find out if the patients did have or did not have Helicobacter pylori before surgery. Results - The final results show that most of the patients had very good and good clinical evolution (Visick I e II) in $96 \%$. The most common symptoms on late postoperative are mild dyspepsia with or without Helicobacter pylori, and diarrhea, anemia and dumping occurred in, respectively, $11(18.6 \%), 2(3.4 \%)$ and $2(3.4 \%)$ cases. The Billroth I reconstruction had the best clinical results on statistical rate. The endoscopic finding showed normal results in the most number of cases, and reflux alkaline gastritis or erosive gastritis in a few cases. Ulcer recurrences were diagnosed in two patients (3.4\%), and both had positive Helicobacter pylori. Most of the patients had Helicobacter pylori (86\%) before surgery and also in the postoperative time (89.9\%). Conclusions - The patients had a very good clinical evolution after the gastrectomy. The Billroth I reconstruction had the best clinical results. The Helicobacter pylori is still present on gastric stump in late postoperative time, and we believe that it does not bring any negative influence to surgical results.

HEADINGS - Peptic ulcer. Gastrectomy. Helicobacter pylori. Postgastrectomy syndromes. 


\section{REFERÊNCIAS BIBLIOGRÁFICAS}

1. Andreollo NA. Contribuição à etiologia do câncer do coto gástrico - Estudo experimental [tese]. Campinas: Universidade Estadual de Campinas; 1994.

2. Andreollo NA, Bratfisch E, Coelho-Neto JS, Lopes LR, Brandalise NA, Leonardi LS. Avaliação pós-operatória tardia de doentes gastrectomizados por úlcera péptica. Rev Bras Med 1999;56:498-515

3. Archimandritis A, Apostolopoulos P, Sougioultzis S, Delladetsima I, Davaris P, Tzivras $\mathrm{M}$. The CLO test is unreliable in diagnosing $H$. pylori infection in post-surgical stomach; is there any hole of $H$. pylori in peptic ulcer recurrence? Eur J Gastroenterol Hepatol 2000;12:93-6.

4. Bahnacy A, Kupcsulik P, Elés Z, Flautner L. Occurrence and significance of Helicobacter pylori infection after gastric surgery. Acta Chir Hung 1995;35:10-7.

5. Boixeda D, Gisbert JP, Rafael L, Redondo C, San Román AL, Argila CM, Cano A Prevalence of Helicobacter pylori infection in gastrectomy and vagotomy. Rev Clin Esp 1995; 195:757-60.

6. Braghetto I, Bosch EH, Csendes A. Diarrea postoperatoria en cirurgía de úlcera péptica. Rev Chil Cir 1986;38:124-9.

7. Coelho JCU, Machi J. Síndromes pós operações gástricas (síndromes pós-gastrectomias). In: Coelho J, editor. Aparelho digestivo - Clínica e cirurgia. Rio de Janeiro: Editora Médica e Científica; 1990. v. 1, p.274-81.

8. Danesh J, Appleby P, Peto R. How often does surgery for peptic ulceration eradicate Helicobacter pylori? Systematic review of 36 studies. BMJ 1998;316:746-7.

9. Delcore R, Cheung LY. Surgical options in postgastrectomy syndromes. Surg Clin North Am 1999;71:57-75.

10. Dixon MF, Genta RM, Yardley JH, Correa P. Classification and grading of gastritis. The updated Sydney System. International Workshop on the Histopathology of Gastritis, Houston 1994. Am J Surg Pathol 1996;20:1161-81.

11. Fukuhara K, Osugi H, Takada N, Takemura M, Lee S, Taguchi S, Kaneko M, Tanaka Y, Fujiwara Y, Nishizawa S, Kinoshita H. Correlation between duodenogastric reflux and remnant gastritis after distal gastrectomy. Hepatogastroenterology 2004;51:1241-4.

12. Gilliam AD, Speake WJ, Lobo DN, Beckingham IJ. Current practice of emergency vagotomy and Helicobacter pylori eradication for complicated peptic ulcer in the United Kingdom. Br J Surg 2003;90:88-90.

13. Goligher JC, Hill GL, Keny TE, Nutter E. Proximal gastric vagotomy without drainage for duodenal ulcer: results after 5-8 years. Br J Surg 1978;65:145-51.

14. Heymann TD, Wilson PD. Helicobacter pylori and surgery. Role of bile reflux is insufficiently explained. BMJ 1998;317:679.

15. Johnston D, Martin I. Duodenal ulcer and peptic ulceration. In: Maingot's. Abdominal operations. $10^{\text {th }}$ ed. Stanford: Appleton \& Lange; 1997; v. 1, p.941-69.

16. Juncosa GJP, Canavesi DC, Montedonico CF. Complicaciones en el tratamiento quirúrgico de la úlcera gastroduodenal. Cir Urug 1994;64:179-85.

17. Karat D, Griffin SM. Helicobacter pylori and surgery. Bile reflux is important in eradicating Helicobacter pylori. BMJ 1998;317:679.

18. Kurata JH, Haile BM, Elashoff JD. Sex differences in peptic ulcer disease. Gastroenterology 1985;88:96-100.

19. Lee YT, Sung JJ, Choi CL, Chang FK, Ng EK, Ching JY, Leung WK, Chung SC. Ulcer recurrence after gastric surgery: is Helicobacter pylori the culprit? Am J Gastroenterol 1998;93:928-31.

20. Lee Y, Tokunaga A, Tajiri T, Masuda G, Okuda T, Fujita I, Kiyama T, Yoshiyuki T, Kato S, Matsukura N, Yamada N. Inflammation of the gastric remnant after gastrectomy: mucosal erythema is associated with bile reflux and inflammatory cellular infiltration is associated with Helicobacter pylori infection. J Gastroenterol 2004;39:520-6.
21. Leivonen M, Nordlin S, Haglund C. The course of Helicobacter pylori infection after gastric parcial gastrectomy for peptic ulcer disease. Hepatogastroenterology 1998;45:587-91.

22. Magalhães AFM. Úlcera péptica. Rev Bras Med 2000;57:1203-12.

23. Mégraud F. Advantages and disadvantages of current diagnostic tests for the detection of Helicobacter pylori. Scand J Gastroenterol 1996;215(Suppl):57-62.

24. Okumura M, Kwang Wt, Bonetti EI, Libonat JR, Lex A. Acidentes e complicações da gastrectomia. Rev Hosp Clin Fac Med São Paulo 1986;41:228-39.

25. Onoda N, Maeda K, Sawada T, Wakasa K, Arakawa T, Chung KH. Prevalence of Helicobacter pylori infection in gastric remnant after distal gastrectomy for primary gastric cancer. Gastric Cancer 2001;4:87-92.

26. Osugi H, Fukuhara K, Takada N, Takemura M, Kinoshita H. Reconstructive procedure after distal gastrectomy to prevent remnant gastritis. Hepatogastroenterology 2004;51:1215-8.

27. Papini-Berto SJ. Desnutrição protéico-energética (DPE) no paciente submetido a gastrectomia. Efeito do critério de classificação nutricional, tipo de cirurgia e tempo pós-operatório sobre a incidência e gravidade da DPE [tese]. São Paulo: Faculdade de Ciências Farmacêuticas, Universidade de São Paulo; 1998.

28. Peterson WL, Fendrick AM, Cave DR, Peura DA, Garabedian-Ruffalo SM, Laine L. Helicobacter pylori - related disease: guidelines for testing and treatment. Arch Intern Med 2000;160:1285-91.

29. Rino Y, Iamada T, Shiozawa M, Takahashi M, Fukuzawa K, Hasuo K, Nagano A Tanaka J, Hatori S, Amano T, Kondo J. Helicobacter pylori of remnant stomach and its eradication. Hepatogastroenterology 1999;46:2069-73.

30. Safatle-Ribeiro AV, Ribeiro Jr U, Sakai P, Iriya K, Ishioka S, Gama-Rodrigues J. Gastric stump mucosa: is there a risk for carcinoma? Arq Gastroenterol 2001:38:227-31.

31. Sawyers JL. Management of postgastrectomy syndromes. Am J Surg 1990;159:8-14

32. Sezin M, Máximo C, Capra A, Gramatica L, Centarti JC. Tratamiento quirúrgico de la úlcera gastroduodenal crónica: 50 años de experiência. Prensa Med Argent 1985;72:398-402.

33. Sheu BS, Lee SC, Lin PW, Wang ST, Chang YC, Yang HB, Cuan CH, Lin XZ. Carbon urea breath test is not as accurate as endoscopy to detect Helicobacter pylori after gastrectomy. Gastrointest Endosc 2000;51:670-5.

34. Soybel D, Zinner MJ. Stomach and duodenum: operative procedures. In: Maingot's Abdominal operations. $10^{\text {th }}$ ed. Stanford: Appleton \& Lange; 1997. v. 1, p.1079-127.

35. Thirlby RC. Postoperative recurrent ulcer. Gastroenterol Clin North Am 1994;23:295-311.

36. Thompson JC, Weiner T. Evaluation of surgical treatment of duodenal ulcer: short and long-term effects. Clin Gastroenterol 1984;13:569-600.

37. Tomtitchong P, Onda M, Matsukura N, Tokunaga A, Kato S, Matsuhita T, Yamada N, Hayashi A. Helicobacter pylori infection in the remnant stomach after gastrectomy: with special reference to the difference between Billroth I and II anastomoses. J Clin Gastroenterol 1998;27:154s-8s

38. Tovey FI, Clark CG, Anaemia after partial gastrectomy: a neglected curable condition Lancet 1980;1:956-8.

39. Tovey FI, Godfrey JE, Lewin MR. A gastrectomy population: 25-30 Years on. Postgrad Med J 1990;66:450-6.

40. Towfigh S, Chandler C, Hines OJ, McFadden DW. Outcomes from peptic ulcer surgery have not benefited from advances in medical therapy. Am Surg 2002;68:385-9.

41. Tytgat GN, Offerhaus GJ, Mulder CJ, vd Berg BT. Consequences of gastric surgery for benign conditions: an overview. Hepatogastroenterology 1988;35:271-8.

42. Visick AH. A study of the failures after gastrectomy. Ann R Coll Surg Engl 1948;3:266-84.

Recebido em 19/8/2004 Aprovado em 14/4/2005 